Article

\title{
Field Efficacy and Transmission of Fast- and Slow-Killing Nucleopolyhedroviruses that Are Infectious to Adoxophyes honmai (Lepidoptera: Tortricidae)
}

\author{
Maho Takahashi ${ }^{1,2}$, Madoka Nakai ${ }^{1, *}$, Yasumasa Saito ${ }^{1}$, Yasushi Sato ${ }^{3}$, Chikara Ishijima ${ }^{3,4}$ \\ and Yasuhisa Kunimi ${ }^{1}$
}

1 United Graduate School of Agricultural Science, Tokyo University of Agriculture and Technology, Fuchu, Tokyo 183-8509, Japan; E-Mails: m.tkhsh188@pref.chiba.lg.jp (M.T.); saitoyassu@gmail.com (Y.S.); kunimi@cc.tuat.ac.jp (Y.K.)

2 Chiba Prefectural Agriculture and Forestry Research Center, Chiba 266-0006, Japan

3 NARO Institute of Vegetable and Tea Science, Shimada, Shizuoka 428-8501, Japan; E-Mails: lucifer@affrc.go.jp (Y.S.); cishiji@affrc.go.jp (C.I.)

4 NARO Agricultural Research Center, 3-1-1 Kannondai, Tsukuba, Ibaraki 305-8666, Japan

* Author to whom correspondence should be addressed; E-Mail: madoka@cc.tuat.ac.jp; Tel./Fax: +81-42-367-5695.

Academic Editor: Karyn Johnson

Received: 6 January 2015 / Accepted: 11 March 2015 / Published: 18 March 2015

\begin{abstract}
The smaller tea tortrix, Adoxophyes honmai (Lepidoptera: Tortricidae), is an economically important pest of tea in Japan. Previous work showed that a fast-killing nucleopolyhedrovirus (NPV) isolated from A. orana (AdorNPV) and a slow-killing NPV isolated from $A$. honmai (AdhoNPV) are both infectious to $A$. honmai larvae. Field application of these different NPVs was conducted against an A. honmai larval population in tea plants, and the control efficacy and transmission rate of the two NPVs were compared. The slow-killing AdhoNPV showed lower field efficacy, in terms of preventing damage caused by $A$. honmai larvae against the tea plants, than the fast-killing AdorNPV. However, AdhoNPV had a significantly higher horizontal transmission rate than AdorNPV. These results show that AdorNPV is suitable as an inundative agent, while AdhoNPV is an appropriate inoculative agent.
\end{abstract}


Keywords: nucleopolyhedrovirus; tea field; killing speed; transmission rate; leaf damage; alternative agent

\section{Introduction}

Adoxophyes honmai (Lepidoptera: Tortricidae), the smaller tea tortrix, is an economically important pest of tea in Japan. A. honmai usually occurs together with the tea tortrix, Homona magnanima (Lepidoptera: Tortricidae), another tea pest of lesser importance in Southern Japan. A. honmai generally has four generations per year, but five in the southern part of Japan [1].

Baculoviruses are a well-known group of insect viruses that have potential as microbial insecticides against pests in agriculture and forestry [2]. The family Baculoviridae comprises the four genera, which comprise Alphabaculovirus (nucleopolyhedrovirus (NPV), infecting lepidopteran host), Betabaculovirus (granulovirus (GV), infecting lepidopteran host), Deltabaculovirus (dipteran NPV), and Gammabaculovirus (hymenopteran NPV). Baculoviruses produce occlusion bodies (OBs), which are structures that embed virus particles in infected host cells and protect them from environmental hazards including ultraviolet light, thus, allowing the occluded virus particles to persist for prolonged periods between their release from an infected host and uptake by a new host. Horizontal transmission of baculoviruses from one host to another occurs when a susceptible insect ingests food contaminated with OBs [3]. A. honmai and H. magnanima are susceptible to Adoxophyes orana GV (AdorGV) and $H$. magnanima $G V$ (HomaGV), respectively. A biopesticide based on both viruses has been registered as the commercial product Hamaki-Tenteki (Arysta LifeScience Corp., Tokyo, Japan). Hamaki-Tenteki is effective against $A$. honmai and $H$. magnanima but its killing speed in $A$. honmai larvae is slow: approximately 40 days are required to kill neonate larvae infected by AdorGV and HomaGV [4,5].

Development of baculovirus resistance in the codling moth, Cydia pomonella (Lepidoptera: Tortricidae), against commercially applied C. pomonella $\mathrm{GV}(\mathrm{CpGV})$ has occurred in Europe [6], but the resistance was overcome by other isolates of $\mathrm{CpGV}$, which have different genotypes [7]. From this point of view, alternative virus isolates should be kept in reserve to sustain prolonged viral control of tea pests in Japan. For this purpose, Japanese isolates of two other viruses infectious to $A$. honmai larvae, A. honmai NPV (AdhoNPV) [8,9], and A. honmai entomopoxvirus (AHEV) [10], are good candidates. A. orana NPV (AdorNPV) was isolated in England and is closely related to AdhoNPV but its killing speed is faster than AdhoNPV [11]. If the biological characteristics of these other viruses are as suitable as AdorGV, they may be useful alternative biocontrol agents. Takahashi et al. (2008) reported the biological characteristics of NPVs infectious to A. honmai larvae [12]. The killing speeds of AdhoNPV (Japanese isolate) and AdorNPV (English isolate) against neonate larvae were 19 and 6 days. On the other hand, the yield of AdhoNPV OBs was significantly higher than that of AdorNPV OBs. However, field studies of these isolates have not yet been conducted.

In this study, we examined two important parameters of AdhoNPV and AdorNPV as biocontrol agents: field control efficacy and viral transmission. The potential of these two slow- and fast-killing NPVs as biocontrol agents against $A$. honmai is discussed. 


\section{Materials and Methods}

\subsection{Insects}

A. honmai used in experiments for speed of kill and cadaver location was obtained from the Agro-Kanesho Co., Ltd. (Tokorozawa, Saitama, Japan). To avoid genetic pollution of local A. honmai population, A. honmai released for the field trial in Shizuoka, were originated from Shimada, Shizuoka Prefecture, Japan. Both insect cultures were maintained in the laboratory at $25{ }^{\circ} \mathrm{C}$, and with a 16 -h light/8-h dark (16L: 8D) photoperiod. Larvae were reared on an artificial diet (Insecta LFS; Nosan Corporation, Yokohama, Kanagawa, Japan).

\subsection{Viruses}

AdhoNPV was originally isolated from diseased larvae collected in a tea field in Tsukuba, Ibaraki, Japan [8,13]. AdorNPV was originally isolated from diseased larvae collected in Kent, England [11]. Both viruses were propagated in $A$. honmai larvae and the OBs were purified as described elsewhere $[9,10]$. For the field trial, viral OBs were semi-purified as follows. Infected $A$. honmai cadavers were homogenized with distilled water. This suspension was filtered through two layers of gauze and centrifuged at $100 \times g$ for $5 \mathrm{~min}$. The supernatant was centrifuged for $20 \mathrm{~min}$ at $3500 \times \mathrm{g}$, and the pellet was suspended in distilled water. The concentration of OBs in this stock suspension was determined by phase-contrast microscopy at $600 \times$ using a bacterial counter (Helber bacteria counting chamber, Hawksley, Sussex, UK), and the OBs were stored at $-18^{\circ} \mathrm{C}$ until used.

\subsection{Killing Speed of AdhoNPV and AdorNPV}

Larval A. honmai were inoculated with AdhoNPV or AdorNPV using a modified droplet feeding method [9]. Neonates and newly molted larvae were allowed to feed on a liquid inoculum containing a dose of $>$ LC 95 of AdhoNPV or AdorNPV OBs, $10 \%(w / v)$ sucrose, and 5\% (w/v) red food coloring. The concentrations of inoculum were $3.0 \times 10^{8} \mathrm{OBs} / \mathrm{mL}$ for neonate, $2^{\text {nd }}$ and $3^{\text {rd }}$ instar, $1.0 \times 10^{8} \mathrm{OBs} / \mathrm{mL}$ for $4^{\text {th }}$ instar and $2.0 \times 10^{9} \mathrm{OBs} / \mathrm{mL}$ for $5^{\text {th }}$ instar. The same solution without virus was used as a mock inoculum in control experiments. Larvae were reared until they died or pupated. The intervals at mortality assessments were made every $24 \mathrm{~h}$.

\subsection{Field Cage Trials}

Field trials were conducted in three insect-proof net cages $(4.5 \times 4.5 \times 2 \mathrm{~m}$ height $)$ that were placed over two rows $(1.5 \times 3.7 \times 0.7 \mathrm{~m}$ height) of tea plants (cv, "Yabukita"), in NARO Institute of Vegetable and Tea Science at Shimada, Shizuoka, Japan $\left(34^{\circ} 48^{\prime} \mathrm{N}, 138^{\circ} 08^{\prime} \mathrm{E}\right)$. These three cages were used for two viral treatments (AdhoNPV and AdorNPV) and a virus-free control treatment.

Thirty egg masses, which had developed to the pharate larval stage (denoted by a change in the surface color to black), were transferred to each cage and attached to young leaves on 22 August 2008. Four days later, $0.2 \mathrm{~L} / \mathrm{m}^{2}$ of OB suspension with $0.01 \%$ spreading agent "Gramin S" (Sankyo Agro, Tokyo, Japan) was applied to each cage using an electric sprayer (BH-593, Matsushita, Tokyo, Japan) with a single nozzle. The OB concentrations of AdhoNPV and AdorNPV were $4.4 \times 10^{9}$ and 
$7.4 \times 10^{8} \mathrm{OBs} / \mathrm{m}^{2}$, which yielded $90 \%$ mortality in a preliminary leaf dipping test. The control treatment cage was sprayed with water containing $0.01 \%$ Gramin S. The experiment was conducted only once.

A. honmai larvae roll tea leaves and damage them in the rolled leaves, which are referred to as "nests" (Figure 1). Usually, a nest is produced by a single larva, and the number of nests is, thus, equivalent to the number of larvae. The number of nests and the size of the damage area on the surface of the leaves were investigated weekly by a quadrat method, at 1-3 weeks after spraying. This method consisted of randomly placing ten $0.5 \times 0.5 \mathrm{~m}$ quadrats on the upper surface of tea plants in each cage on each assessment day, and recording the number of nests and the damage area of leaves in each square. The damage areas were categorized as "Small $\left(<25 \mathrm{~mm}^{2}\right)$ ", "Medium $\left(25-100 \mathrm{~mm}^{2}\right)$ ", "Large (100-400 mm $\mathrm{mm}^{2}$ ", or "Extra-large $\left(>400 \mathrm{~mm}^{2}\right)$ ".

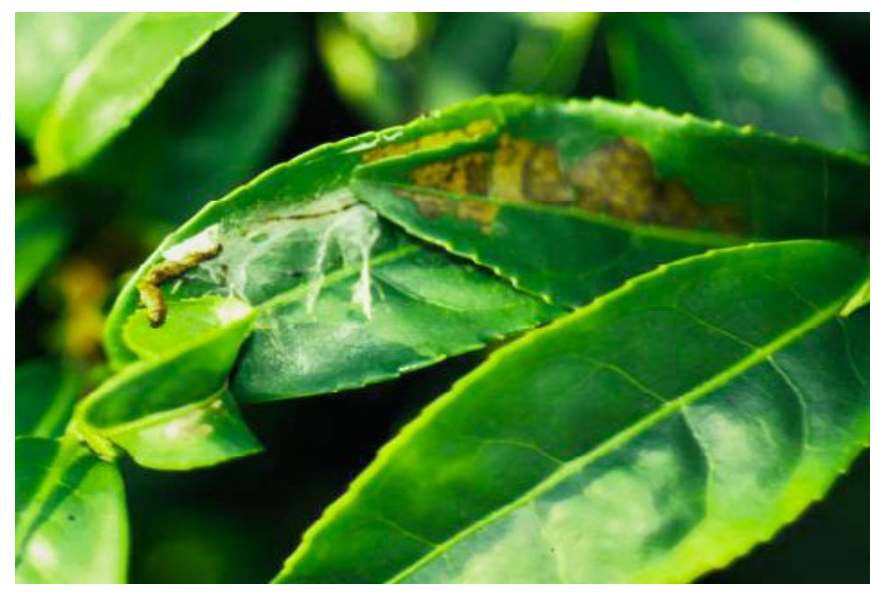

Figure 1. Rolled leaves, referred to as nests of $A$. honmai larvae. In the nest the larva damages the leaf. In this picture a cadaver is located outside its nest.

At days after treatment, A. honmai $2^{\text {nd }}$ instar larvae were released into each cage to examine viral transmission to the next generation. The release timing of second generation was supposed as the timing of next generation of healthy A. honmai survived from viral application and/or migrated from other tea fields. Second release was done once and the timing (29 September 2008) was selected on the basis of data for developmental zero and thermal constants of healthy A. honmai [14]. Three hundred eighty larvae were released on four spots in each cage (1520 larvae per cage). Four days later, the released larvae were collected from each cage; these larvae were $2^{\text {nd }}$ or $3^{\text {rd }}$ instars. Larvae were transferred individually into 20 -mL cups containing fresh artificial diet (Insecta LFS) and kept at $25{ }^{\circ} \mathrm{C}$ and a $16 \mathrm{~L}$ : $8 \mathrm{D}$ photoperiod in an incubator. Larvae were reared until they died or pupated. Dead insects were diagnosed as virus-infected if OBs were visible in tissue smears under a phase-contrast microscope.

\subsection{Position of Infected Insect Cadavers}

Virus suspensions were prepared with OBs of AdhoNPV $\left(2.2 \times 10^{7} \mathrm{OBs} / \mathrm{mL}\right)$ and AdorNPV $\left(3.7 \times 10^{6} \mathrm{OBs} / \mathrm{mL}\right)$, equivalent to $\mathrm{LC}_{90}$ of neonate larvae in the droplet feeding method [12], and a final concentration of $0.02 \%$ of the spreading agent. Twenty milliliters of virus suspension was sprayed onto a branch of a tea plant with 30-40 leaves in Tokyo University of Agriculture and Technology in Fuchu, Tokyo, Japan $\left(35^{\circ} 41^{\prime} \mathrm{N}, 139^{\circ} 29^{\prime} \mathrm{E}\right)$, which was then completely covered with a polyester net 
(22 cm diameter, $33 \mathrm{~cm}$ length, $1 \mathrm{~mm}$ mesh). A control branch was sprayed with $0.02 \%$ spreading agent. Spraying was done in the evening to avoid inactivation by sunlight, and each treatment was repeated six times. Egg masses of A. honmai were stapled to the underside of leaves, after the leaves were air-dried. Infected cadavers were collected 10 and 31 days after virus application for AdorNPV and AdhoNPV, respectively, and the number of insects that died at each of three positions (inside or outside rolled leaves, or having fallen off) was counted. NPV infection was confirmed by light microscopy.

\subsection{Statistical Analysis}

The time taken by viruses to kill the larvae was analyzed by nonparametric comparisons for two pairs using the Wilcoxon Test. The number of nests was $\log (\mathrm{x}+1)$ transformed and analyzed using analysis of variance (ANOVA). When there was a significant difference among treatments, Tukey-Kramer multiple comparison tests were performed $(\alpha=0.05)$. Transmission rate and cadaver location data were arcsin square-root transformed and analyzed by $t$-test.

\section{Results and Discussion}

\subsection{Killing Speed}

Figure 2 shows mortality and developmental time of AdhoNPV- and AdorNPV-infected A. honmai. AdhoNPV-infected larvae died only at the final instar regardless at which instar they were inoculated, and the mean survival time \pm standard error (SE) was $18.9 \pm 0.6,15.2 \pm 0.4,12.2 \pm 0.2,10.1 \pm 0.2$, and $8.0 \pm 0.1$ days for neonate, $2^{\text {nd }}, 3^{\text {rd }}, 4^{\text {th }}$ and $5^{\text {th }}$ instar inoculation, respectively. On the other hand, the mean survival time $\pm \mathrm{SE}$ of AdorNPV-infected larvae was $5.8 \pm 0.1,7.0 \pm 0.1,7.0 \pm 0.1,8.0 \pm 0.1$, and $6.9 \pm 0.1$ days for neonate, $2^{\text {nd }}, 3^{\text {rd }}, 4^{\text {th }}$ and $5^{\text {th }}$ instar inoculation, respectively. Death caused by AdhoNPV infection was significantly slower than that by AdorNPV for any time of inoculation $\left(1^{\text {st }}\right.$ inoculation: $H=39.17, p<0.01 ; 2^{\text {nd }}$ inoculation: $H=55.40, p<0.01 ; 3^{\text {rd }}$ inoculation: $H=65.72, p<0.01$; $4^{\text {th }}$ inoculation: $H=55.03, p<0.01$; and $5^{\text {th }}$ inoculation: $H=21.02, p<0.01$ ).

The genomes of AdhoNPV and AdorNPV have been fully sequenced and compared with each other [11,13]. Among 121 putative ORFs, 118 are homologous to ORFs in AdhoNPV genome with $83 \%-100 \%$ amino acid identity. The main difference between them is partial deletion of ecdysteroid UDP-glucosyltransferase (egt) for AdorNPV compared to full possession of egt for AdhoNPV; however, genes related to killing speed of these viruses have not yet been determined. Differences in killing speed among closely related baculovirus genotypes have also been addressed in other baculovirus species. For instance, field isolates of Agrotis ipsilon NPV consist of isolates with and without an egt gene, and egt-deleted isolates showed decreased pathogenicity and increased killing speed [15]. Spodoptera frugiperda multiple NPV field isolates also include a fast-killing genotype that displays a deletion of ORF $s f 27$ and egt. Further study is needed to confirm egt or the other gene(s) involved in different killing speeds for AdhoNPV and AdorNPV. The two isolates were used to examine further whether killing speed of field isolates affects efficacy and viral transmission rate. 

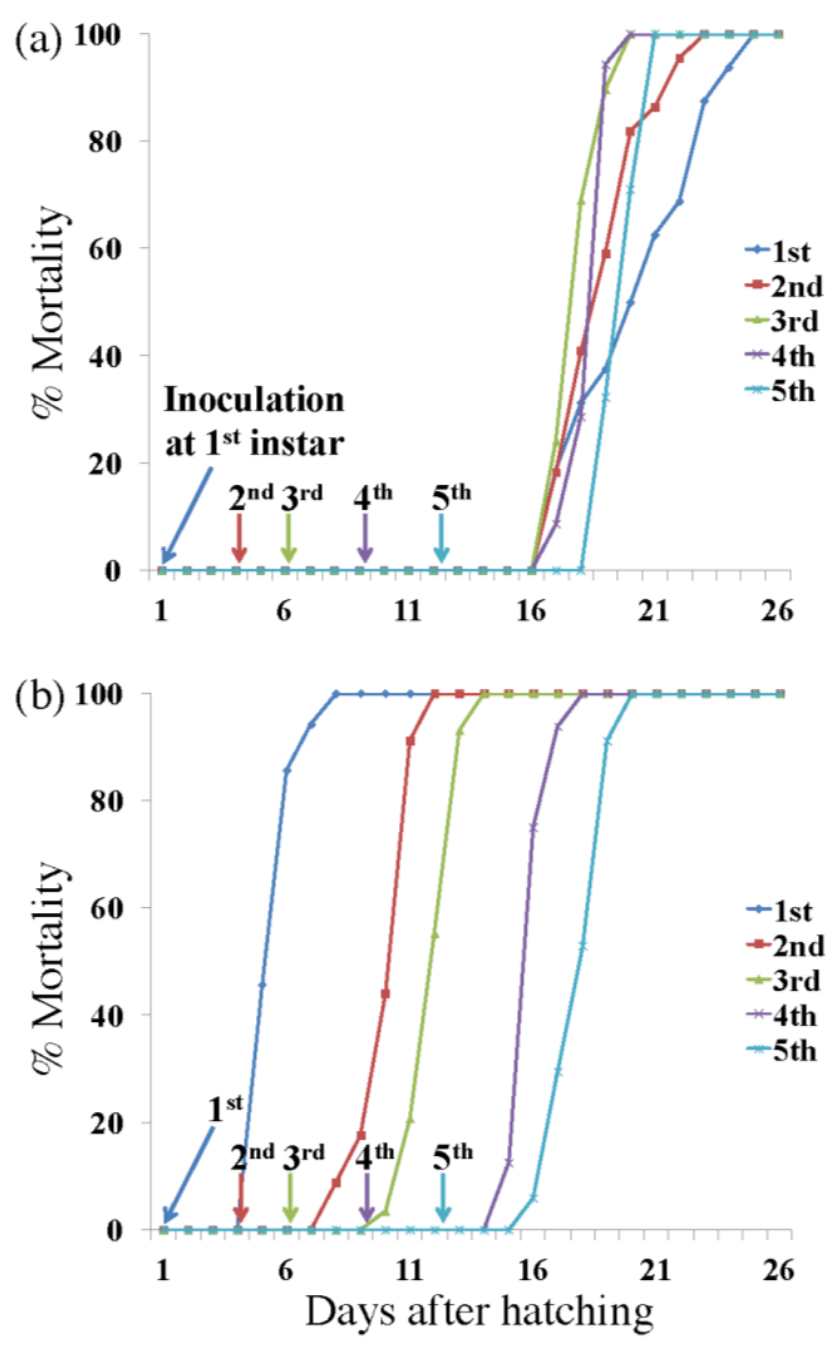

Figure 2. (a) Percentage mortality of $A$. honmai larvae inoculated with AdhoNPV at $1^{\text {st }}, 2^{\text {nd }}$, $3^{\text {rd }}, 4^{\text {th }}$ and $5^{\text {th }}$ instar; (b) Percentage mortality of $A$. honmai larvae inoculated with AdorNPV at $1^{\text {st }}, 2^{\text {nd }}, 3^{\text {rd }}, 4^{\text {th }}$ and $5^{\text {th }}$ instar.

\subsection{Field Efficacy within Sprayed Populations}

AdorNPV showed greater potential than AdhoNPV for faster suppression of an A. honmai population. The mean numbers of $A$. honmai nests per quadrat for AdorNPV treatment were significantly lower than those for other treatments at two weeks $(F=8.47 ; d f=2,27 ; p<0.01)$ and three weeks after virus application $(F=10.79 ; d f=2,27 ; p<0.01)$, whereas there were no significant differences at one week $(F=0.09 ; d f=2,27 ; p=0.91)$ (Figure 3). 


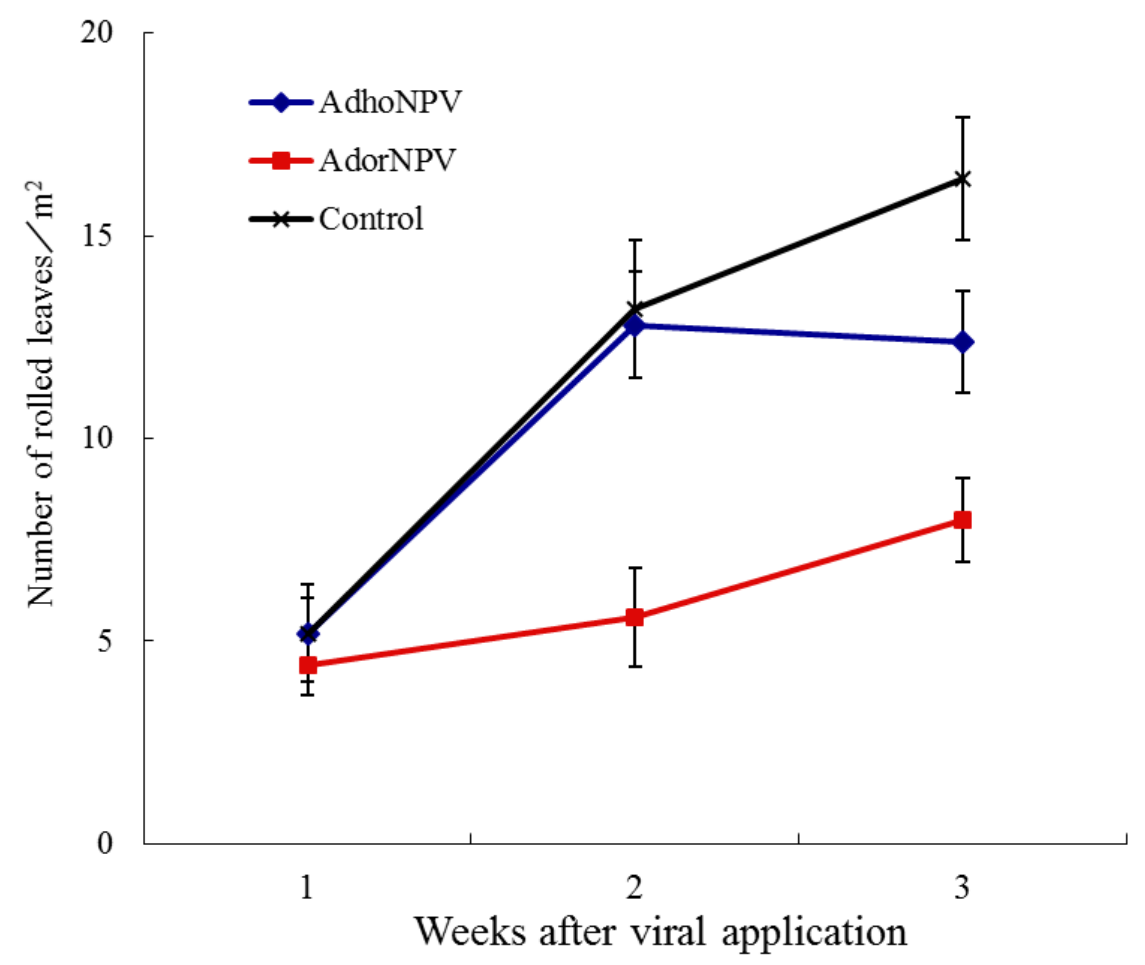

Figure 3. Number of rolled tea leaves produced by $A$. honmai larvae on tea plants in cages sprayed with AdhoNPV, AdorNPV, or virus-free control. Bars indicate standard errors.

Damage areas in the treated plots were categorized to "Small", "Medium", "Large" and "Extra-Large" and the number of nests with damaged area were shown in Figure 4. The number of nests was less at 1 week because only visible nests were counted, and no significant difference was observed for "Small" and "Medium" damages among the treatment (Small, $F=0.0018, d f=2,27, p=0.998$; Medium, $F=0.00, d f=2,27, p=1)$. At two weeks, more nests with "Large" damage were observed in the control and AdhoNPV-treatment than in AdorNPV, which was significantly different, as well as the number of "Medium" damages (Medium, $F=4.22, d f=2,27, p=0.0025$; Large, $F=3.48, d f=2,27$, $p=0.045)$. At three weeks, damage areas in the control and AdhoNPV-treated cages were more than those in AdorNPV-treated cage. The number of nests in the "Large" and "Extra-large" categories in the control and AdhoNPV treatments were more than $50 \%$ of the total. In contrast, most of the nests in the AdorNPV treatment were "Small" (30\%) and "Medium" (50\%) (Figure 4). Number of leaves with "Small", "Medium", or "Large" damage were not significantly different among the three treatment ("Small", $F=0.012, d f=2,27, p=0.98$; "Medium", $F=0.456, d f=2,27, p=0.64$; "Large", $F=2.25$, $d f=2,27, p=0.13$ ), but number of leaves with "Extra-large" damage was significantly different $(F=11.80, d f=2,27, p=0.0002)$. Tukey-Kramer multiple comparison among the three treatments revealed that number of leaves with "Extra-Large" damage in control was significantly more than that in either AdhoNPV or AdorNPV $(p<0.05)$. Even though no significant difference was detected, number of leaves with "Extra-Large" damage was six-fold less in AdorNPV than in AdhoNPV $(p>0.05)$. These results were in agreement with previous laboratory bioassays [12]. 


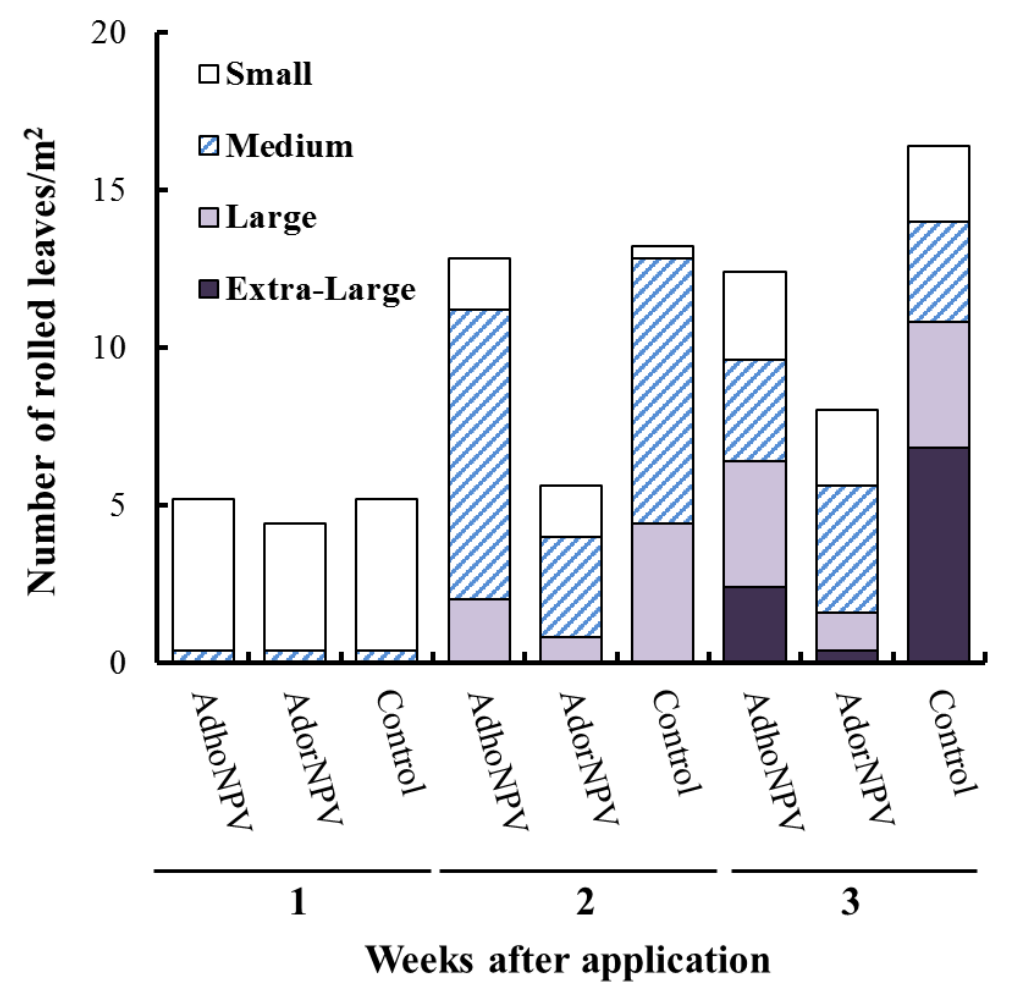

Figure 4. Number of rolled leaves produced by $A$. honmai larvae with damaged area. Damaged areas on the leaves were categorized as "Small $\left(<25 \mathrm{~mm}^{2}\right)$ ", "Medium (25-100 mm²)", "Large (100-400 $\left.\mathrm{mm}^{2}\right)$ ", or "Extra-large $\left(>400 \mathrm{~mm}^{2}\right)$ ".

The difference in efficacy between AdorNPV and AdhoNPV was probably due to the major difference in killing speed: the mean survival time of AdorNPV-infected larvae was six days, 13 days shorter than AdhoNPV-infected larvae (mean survival time: 19 days) when A. honmai larvae were inoculated at first instar (Figure 2). Speed of kill is the most important characteristic for insecticidal efficacy in the context of preventing crop damage.

Several baculoviruses have already been genetically engineered to improve their killing speed, a notable example being the insertion of an insect-specific toxin gene (AaIT) derived from the scorpion Androctonus australis (Scorpiones: Buthidae) into the baculovirus genome [2,16,17], and their superior efficacy has also been demonstrated in field trials [18-20]. Our results indicated that the fast-killing AdorNPV had superior potential to prevent damage to tea leaves, in comparison to the slow-killing AdhoNPV, against an A. honmai population during the generation in which the virus is applied.

\subsection{Viral Transmission between Generations}

The transmission rate of AdhoNPV to A. honmai larvae was calculated as the percentage infection of larvae released 34 days after viral application, a time which is equivalent to the emergence of the next generation, and was approximately four-fold higher than that of AdorNPV (mean mortality of larvae recovered from AdhoNPV-treated cage $=9.80 \pm 2.46$; of those from AdorNPV-treated cage $=2.52 \pm 1.48 ; t=2.601 ; d f=6, p=0.0406$ ) (Figure 5). No viral transmission occurred in the control cage. No replication for viral application was done for this experiment. The difference between AdhoNPV and AdorNPV transmission may arise from variation in OB production for the two viruses: 
the yield of AdhoNPV OBs per larva $\left(7.0 \times 10^{9}\right)$ was 1,000 - to 10,000-fold higher than that of AdorNPV OBs $\left(5.6 \times 10^{5}-8.7 \times 10^{6}\right.$; depending on instar at death) when neonate larvae were inoculated [12]. The amount of $\mathrm{OB}$ in a host habitat plays an important role in transmission among hosts [21,22].

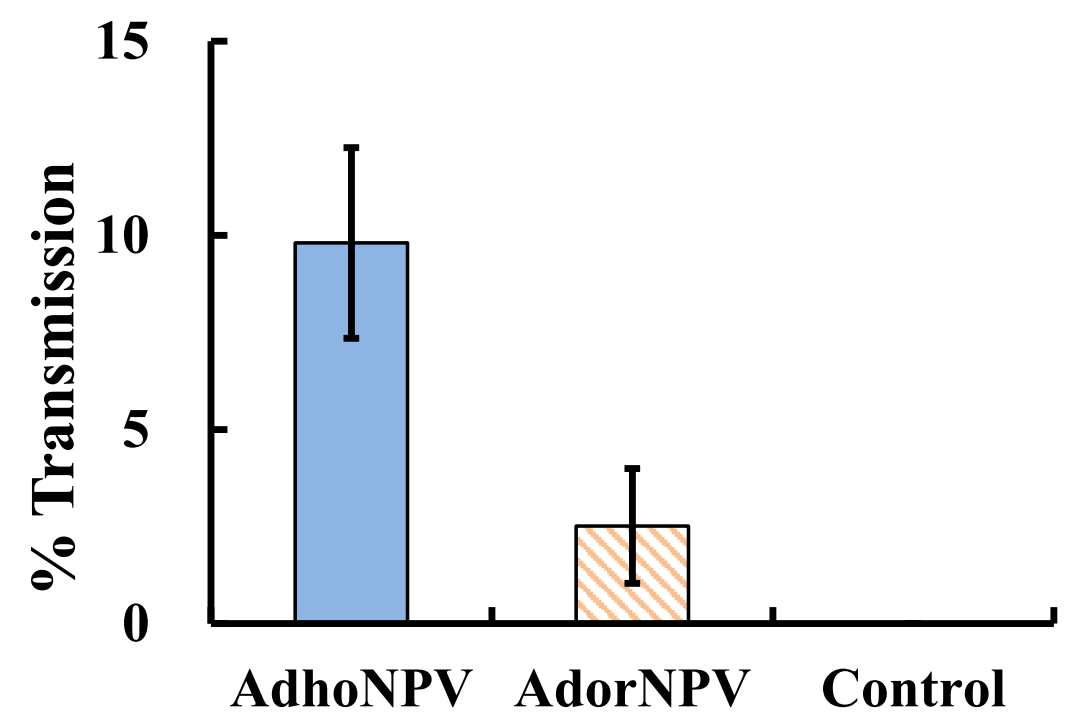

Figure 5. Transmission rate of AdhoNPV and AdorNPV to $A$. honmai larvae in the next generation. Percentage mortality of $A$. honmai larvae released at 34 days after spraying AdhoNPV and AdorNPV on tea leaves. A solution of spreading agent without virus was sprayed as the control. Bars indicate standard errors.

Baculovirus infection is known to alter insect behavior, and several viral genes are implicated in "tree top disease" or "Wipfelkrankheit", in which infected insects climb up the tree canopy and die near the top [23]. This phenotype is adapted for baculoviruses to increase their rate of transmission to other individual hosts. One of the genes involved in this behavior alteration caused by baculovirus infection is egt, because Lymantria dispar (Lepidoptera: Erebidae) larvae infected with an egt deletion mutant of L. dispar NPV climb less high than those infected with wild type virus [24]. Other genes and mechanisms also appear to be involved in this phenotype [25-28]. AdorNPV lacks functional domains in egt, whereas AdhoNPV possesses intact egt. However, both AdhoNPV- and AdorNPV-infected larvae died in their nest (Figure 6): 83.8\% and 81.3\% of cadavers killed by AdhoNPV and AdorNPV, respectively, were located inside nests on the leaves. There was no significant difference, for any position of infected insect cadavers, between AdhoNPV and AdorNPV ( $p>0.05)$. Thus, the difference in transmission rate in our results was not attributable to the locations of infected cadavers, and the difference in OB production is likely to be a factor that drives the difference in transmission rates between the two isolates.

Hochberg (1989) categorized the location of virus-infected cadavers as either a "protected stage" or a "transmissible stage" [22]. According to a study of AaIT expressed by recombinant Autographa californica multiple NPV (AcMNPV), the transmission rate of the fast-killing recombinant virus is lower than that of the wild type AcMNPV, because the yield of recombinant virus was lower than that of wild type and because recombinant-infected larvae fell onto the soil before death [18,29]. Liquefaction of infected cadavers is known to enhance horizontal transmission of NPVs and the v-cath and chi genes, encoding cathepsin and chitinase, respectively, are involved in this process [30-32]. However, the 
AdhoNPV and AdorNPV genomes do not possess a chi gene, and infected cadavers do not liquefy, in contrast to other NPVs including AcMNPV [11,13]. Thus, progeny OBs from both AdhoNPV- and AdorNPV-infected larvae are in the "protected stage" until the cadavers are degraded by desiccation and spread to the tea leaf surface. Meanwhile, the rolled-leaf nests produced by A. honmai larvae may function as a reservoir of OBs, protecting the OBs from direct UV exposure until neonates (the most susceptible stage) of the subsequent generation arrive and are efficiently infected with virus. As mentioned previously, A. honmai have four to five discrete generations per year in Japan, and a slow-killing phenotype, such as AdhoNPV, may be more successfully transmitted to the next generation than a fast-killing phenotype. Other viruses that are infectious for $A$. honmai including AdorGV and AHEV also have a slow-killing phenotype, and the slow-killing trait in these pathogens may, therefore, be convergently adapted to the leaf roller system.

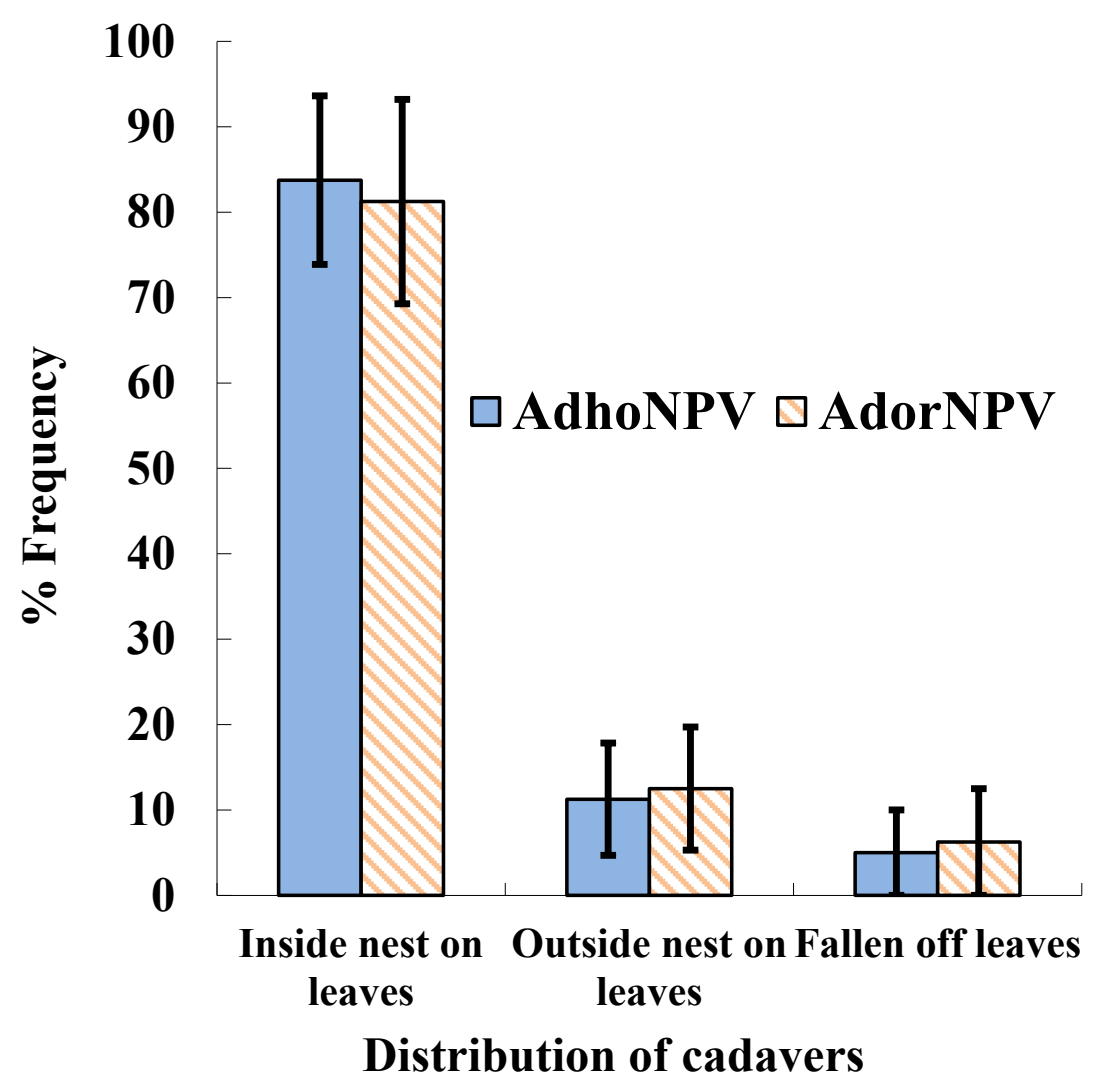

Figure 6. Location of A. honmai cadavers killed by AdhoNPV and AdorNPV on tea leaves. Bars indicate standard errors

\section{Conclusions}

The results of this study revealed that the fast-killing isolate AdorNPV led to less damage to tea leaves than the slow-killing isolate AdhoNPV, since the slow-killing isolate showed a higher transmission rate than the fast-killing isolate; this is because the slow-killing isolate produced more progeny OBs than the fast-killing isolate. There are four main approaches used in microbial control of insects: the short-term microbial insecticide approach known as "inundative release", the recycling microbial insecticide approach of "inoculative release", the introduction-establishment approach, and environmental 
manipulation [33]. Takahashi et al. (2008) proposed that AdorNPV is appropriate for inundative release, while AdhoNPV is appropriate for inoculative release [12]. This study has demonstrated that the fast-killing AdorNPV controls pests more efficiently in the short term than the slow-killing AdhoNPV because of its higher field efficacy against pests within the generation exposed to virus. On the other hand, the slow-killing AdhoNPV controls pests more effectively than fast killing AdorNPV in the long term because of its higher transmission efficiency. AdhoNPV may have an advantage in field efficacy against the sprayed population compared to the commercial agent Hamaki-Tenteki, which includes AdorGV, because the killing speed of AdhoNPV is faster than that of AdorGV. Further field experiments on a larger scale are necessary to evaluate the long-term control efficiency of AdhoNPV as a candidate alternative control agent to AdorGV.

\section{Acknowledgments}

This work was partially supported by a grant for Development of new biorational techniques for sustainable agriculture from the Ministry of Agriculture, Forestry, and Fisheries of Japan, and by the Grant-in-Aid for Scientific Research (KAKENHI) Scientific Research (B), Japan Society for the Promotion of Science (JSPS) 18380038. Authors appreciate Doreen Winstanley for the English isolate of AdorNPV, Ian Smith for critical review of this manuscript and Ms. Maki Goto for providing bioassay data.

\section{Author Contributions}

Maho Takahashi, Yasuhisa Kunimi and Madoka Nakai designed and conducted the field experiments. Yasushi Sato and Chikara Ishijima contributed to the field experiment in Shimada, Shizuoka, Japan. Yasumasa Saito did the laboratory experiment for speed of kill. Maho Takahashi and Madoka Nakai wrote the manuscript.

\section{Conflicts of Interest}

The authors declare no conflict of interest.

\section{References}

1. Tamaki, Y. Tortircids in tea. In Tortricid Pests, Their Biology, Natural Enemies and Control; Van der Geest, L.P.S., Evenhuis, H.H., Eds.; Elsevier: Amsterdam, The Netherland, 1991; pp. 541-551.

2. Szewczyk, B.; Hoyos-Carvajal, L.; Paluszek, M.; Skrzecz, W.; de Souza, M.L. Baculoviruses-Re-emerging biopesticides. Biotechnol. Adv. 2006, 24, 143-160.

3. Cory, J.S.; Myers, J.H. The ecology and evolution of insect baculoviruses. Annu. Rev. Ecol. Evol. Syst. 2003, 34, 239-272.

4. Yamada, H.; Oho, N. A granulosis virus, possible biological agent for control of Adoxophyes orana (Lepidoptera: Tortricidae) in apple orchards: I. Mass production. J. Invertebr. Pathol. 1973, 21, 144-148. 
5. Sato, T.; Oho, N.; Kodomari, S. A granulosis virus of the tea tortrix, Homon magnanima DIAKNOFF (Lepidoptera: Tortricidae); Its pathogenicity and Mass-Production method. Appl. Entomol. Zool. 1980, 15, 409-415.

6. Asser-Kaiser, S.; Fritsch, E.; Undorf-Spahn, K.; Kienzle, J.; Eberle, K.E.; Gund, N.A.; Reineke, A.; Zebitz, C.P.W.; Heckel, D.G.; Huber, J.; et al. Rapid emergence of baculovirus resistance in codling moth due to dominant, sex-linked inheritance. Science 2007, 317, 1916-1918.

7. Gebhardt, M.M.; Eberle, K.E.; Radtke, P.; Jehle, J.A. Baculovirus resistance in codling moth is virus isolate-dependent and the consequence of a mutation in viral gene pe38. Proc. Natl. Acad. Sci. USA 2014, 111, 15711-15716.

8. Nakai, M.; Takeda, M.; Kunimi, Y. Seasonal changes in prevalence of viral disease and parasitism by parasitic insects in a larval population of the smaller tea tortrix, Adoxophyes sp. (Lepidoptera: Tortricidae) in a tea field. Appl. Entomol. Zool. 1997, 32, 609-615.

9. Ishii, T.; Nakai, M.; Okuno, S.; Takatsuka, J.; Kunimi, Y. Characterization of Adoxophyeshonmai single-nucleocapsid nucleopolyhedrovirus: Morphology, structure, and effects on larvae. J. Invertebr. Pathol. 2003, 83, 206-214.

10. Takatsuka, J.; Okuno, S.; Ishii, T.; Nakai, M.; Kunimi, Y. Fitness-related traits of entomopoxviruses isolated from Adoxophyes honmai (Lepidoptera: Tortricidae) at three locations in Japan. J. Invertebr. Pathol. 2010, 105, 121-131.

11. Hilton, S.; Winstanley, D. Genomic sequence and biological characterization of a nucleopolyhedrovirus isolated from the summer fruit tortrix, Adoxophyes orana. J. Gen. Virol. 2008, 89, 2898-2908.

12. Takahashi, M.; Nakai, M.; Nakanishi, K.; Sato, T.; Hilton, S.; Winstanley, D.; Kunimi, Y. Genetic and biological comparisons of four nucleopolyhedrovirus isolates that are infectious to Adoxophyes honmai (Lepidoptera: Tortricidae). Biol. Control 2008, 46, 542-546.

13. Nakai, M.; Goto, C.; Kang, W.; Shikata, M.; Luque, T.; Kunimi, Y. Genome sequence and organization of a nucleopolyhedrovirus isolated from the smaller tea tortrix, Adoxophyes honmai. Virology 2003, 316, 171-183.

14. Nabeta, F.H.; Nakai, M.; Kunimi, Y. Effects of temperature and photoperiod on the development and reproduction of Adoxophyes honmai (Lepidoptera: Tortricidae). Appl. Entomol. Zool. 2005, 40, 231-238.

15. Harrison, R.L. Genomic sequence analysis of the Illinois strain of the Agrotis ipsilon multiple nucleopolyhedrovirus. Virus Genes 2009, 38, 155-170.

16. Bonning, B.C.; Hammock, B.D. Development of recombinant baculoviruses for insect control. Annu. Rev. Entomol. 1996, 41, 191-210.

17. Bonning, B.C.; Boughton, A.J.; Jin, H.L.; Harrison, R.L. Genetic enhancement of baculovirus insecticides. In Advances in Microbial Control of Insect Pests; Upadhyay, R.K., Ed.; Kluwer Academic/Plenum Publishers: New York, NY, USA, 2003; pp. 109-126.

18. Cory, J.S.; Hirst, M.L.; Williams, T.; Hails, R.S.; Goulson, D.; Green, B.M.; Carty, T.M.; Possee, R.D.; Cayley, P.J.; Bishop, D.H.L.; et al. Field trial of a genetically improved baculovirus insecticide. Nature 1994, 370, 138-140. 
19. Hoover, K.; Schultz, C.M.; Lane, S.S.; Bonning, B.C.; Duffey, S.S.; Mccutchen, B.F.; Hammock, B.D. Reduction in damage to cotton plants by a recombinant baculovirus that knocks moribund larvae of Heliothis virescens off the plant. Biol. Control 1995, 5, 419-426.

20. Inceoglu, A.B.; Kamita, S.G.; Hammock, B.D. Genetically modified baculoviruses: A historical overview and future outlook. Adv. Virus Res. 2006, 68, 323-360.

21. Anderson, R.M.; May, R.M. The population dynamics of microparasites and their invertebrate hosts. Philos. Trans. R. Soc. B Biol. Sci. 1981, 291, 451-524.

22. Hochberg, M.E. The potential role of pathogens in biological control. Nature 1989, 337, 262-265.

23. Goulson, D. Wipfelkrankheit: Modification of host behavior during baculoviral infection. Oecologia 1997, 109, 219-228.

24. Hoover, K.; Grove, M.; Gardner, M.; Hughes, D.P.; McNeil, J.; Slavicek, J. A gene for an extended phenotype. Science 2011, 333, 1401.

25. Katsuma, S.; Koyano, Y.; Kang, W.; Kokusho, R.; Kamita, S.G.; Shimada, T. The baculovirus uses a captured host phosphatase to induce enhanced locomotory activity in host caterpillars. PLoS Pathog. 2012, 8, e1002644.

26. Kamita, S.G.; Nagasaka, K.; Chua, J.W.; Shimada, T.; Mita, K.; Kobayashi, M.; Maeda, S.; Hammock, B.D. A baculovirus-encoded protein tyrosine phosphatase gene induces enhanced locomotory activity in a lepidopteran host. Proc. Natl. Acad. Sci. USA 2005, 102, 2584-2589.

27. Van Houte, S.; Ros, V.I.D.; Mastenbroek, T.G.; Vendrig, N.J.; Hoover, K.; Spitzen, J.; van Oers, M.M. Protein tyrosine phosphatase-induced hyperactivity is a conserved strategy of a subset of baculoviruses to manipulate lepidopteran host behavior. PLoS One 2012, 7, e46933.

28. Ros, V.I.D.; van Houte, S.; Hemerik, L.; van Oers, M.M. Baculovirus-induced tree-top disease: How extended is the role of egt as a gene for the extended phenotype? Mol. Ecol. 2014, 28, doi:10.1111/mec.13019.

29. Lee, Y.; Fuxa, J.R.; Inceoglu, A.B.; Alaniz, S.A.; Richter, A.R.; Reilly, L.M.; Hammock, B.D. Competition between wild-type and recombinant nucleopolyhedroviruses in a greenhouse microcosm. Biol. Control 2001, 20, 84-93.

30. Burand, J.P.; Nakai, M.; Smith, I. Host-Virus Interactions. In Insect Pathogens, Molecular Approaches and Techniques; Stock, S.P., Vandenberg, J., Glazer, I., Boemare, N., Eds.; CAB International: Oxfordshire, UK, 2009; pp. 195-222.

31. Hawtin, R.E.; Zarkowska, T.; Arnold, K.; Thomas, C.J.; Gooday, G.W.; King, L.A.; Kuzio, J.A.; Possee, R.D. Liquefaction of Autographa californica nucleopolyhedrovirus-infected insects is dependent on the integrity of virus-encoded chitinase and cathepsin genes. Virology 1997, 238, 243-253.

32. D’Amico, V.; Slavicek, J.; Podgwaite, J.D.; Webb, R.; Fuester, R.; Peiffer, R.A. Deletion of $v$-chiA from a baculovirus reduces horizontal transmission in the field. Appl. Environ. Microbiol. 2013, 79, 4056-4064.

33. Fuxa, J.R. Ecology of insect nucleopolyhedroviruses. Agric. Ecosyst. Environ. 2004, 103, $27-43$.

(C) 2015 by the authors; licensee MDPI, Basel, Switzerland. This article is an open access article distributed under the terms and conditions of the Creative Commons Attribution license (http://creativecommons.org/licenses/by/4.0/). 